\title{
Purely electronic zero-phonon line as the foundation stone for high-resolution matrix spectroscopy, single-impurity-molecule spectroscopy, and persistent spectral hole burning. Recent developments
}

\author{
Karl K. Rebane \\ Institute of Physics, University of Tartu, 142 Riia, Tartu, 51014, Estonia \\ E-mail: rebanek@fi.tartu.ee
}

\begin{abstract}
A few examples of recent progress in the study and applications of purely electronic zerophonon line (ZPL) and its offshoots are briefly considered: new experimental values of the narrowest ZPL; time-and-space-domain holography in the femtosecond domain, and the realization of a femtosecond Taffoli gate by it; single-impurity-molecule spectroscopy, its relation to single-photon interference and to the realization of quantum computing; the promises of quantum computing compared to what has already been done in holography.
\end{abstract}

PACS: 33.70.Jg

The purely electronic zero-phonon line (ZPL) is a remarkable feature of low-temperature spectra of the absorption and luminescence of quite a number of various impurity centers in various solid hosts (see [1-5] and references therein). ZPLs can be very narrow and have very high peak absorption cross sections. These valuable properties, as well as several other features, are in close correspondence with features of the Mössbauer $\gamma$-resonance line. Because of this correlation, originating from the symmetry of the harmonic oscillator Hamiltonian in coordinate and momenta [6], J.F. Gross called the ZPL the optical analog of the Mössbauer line [7], helping considerably to popularize optical ZPLs.

I must note that two main features of the optical ZPL were pointed out in [8], five years before the first publication by Mössbauer [9]. That result of this early paper, however, was not given due attention.

ZPLs, as sharp and intense features in the low-temperature spectra of some impurities in solids (without detailed explanation of their properties), were found and fruitfully studied experimentally years before the Mössbauer effect. ZPL widths of around $1 \mathrm{~cm}^{-1}$ were measured in the spectra of rare-earth impurity ions introduced in proper ionic single-crystal hosts and also ruby (see $[10,11]$ and references therein). The new wave of theoretical studies brought the understanding that even the very narrow widths of around $1 \mathrm{~cm}^{-1}$ are essentially inhomogeneous widths $[1,2,5,12,13]$. It was predicted that for dipole-allowed transitions the homogeneous lines should be, at liquid helium temperatures, another 3-4 orders of magnitude narrower still, i.e., the homogeneous linewidth is $10^{-3}$ $-10^{-4} \mathrm{~cm}^{-1} \approx 10-100 \mathrm{MHz}$ (see [5,12-14] and references therein), and even a few orders of magnitude narrower yet for forbidden ones. The theoretical point, later confirmed experimentally [12,14] was that the ZPL's homogeneous width, $\Gamma_{\text {hom }}(T)$, tends in the limit $T \rightarrow 0$ to the value determined by the lifetime of the excited electronic state.

This theoretical prediction stimulated experimentalists to search for methods of how to eliminate or use the inhomogeneous broadening and to utilize the precious properties of ZPLs in the frequency domain.

ZPL became the foundation stone for three novel fields of modern optics and spectroscopy [3,4,15]: (1) very high spectral resolution ZPL studies of atoms and molecules as impurities in low-temperature solids (very high spectral resolution matrix isolation spectroscopy); (2) single impurity molecule spectroscopy (SMS) (see [16] and references therein); (3) persistent spectral hole burning (PSHB) (see [17] and references therein). 


\section{Very high resolution ZPL spectroscopy of impurity centers}

Recently photon echo measurements of optical dephasing of forbidden transitions in rare-earth ions have revealed low-temperature linewidths as narrow as a few tens of hertz. The narrowest features reported up to now is $\Gamma_{\text {bom }} \approx 50 \mathrm{~Hz}$ for the ${ }^{4} I_{15 / 2} \leftrightarrow{ }^{4} I_{13 / 2}$ transition in $\mathrm{Er}^{3+}: \mathrm{Y}_{2} \mathrm{SiO}_{5}$ with $0.001 \%$ concentration of $\mathrm{Eu}^{3+}$ at $T=1.5 \mathrm{~K}$ and in a magnetic field of $B=$ $=70 \mathrm{kG}$ (to freeze the nuclear spin fluctuation, which can be considered as causing unresolved or time-dependent inhomogeneous broadening) $[18,19]$.

In the conventional optical spectroscopy of impurity solids, we have been accustomed to routinely neglecting the effect of the recoil momentum associated with the absorption and emission of optical photons, because the recoil of the emitting/absorbing atom corresponds to a frequency shift of about $1 \mathrm{kHz}$, which is really small compared to the allowed transitions' SMS linewidths about $10-100 \mathrm{MHz}$. For the very narrow ZPLs like those mentioned above, $\Gamma_{\text {hom }}<1 \mathrm{kHz}$, and the recoil has to be taken into account. The development of a new version of the theory of ZPLs taking into account simultaneously the Stokes shift and recoil has been started [20].

A novel aspect is that recoil entangles the emitted photon and the free atom which had emitted it. The entanglement is utilized to build a new quantum description of the spontaneously emitted single localized photon.

The situation for an impurity in a solid is more sophisticated than for an atom in vacuum. ZPL occupies here a very specific position: for ZPL a macroscopic piece of solid accepts and absorbs the recoil, and the recoil momentum and energy received is practically zero. The transitions creating the phonon sideband distribute the recoil between phonons, and the entanglement is not clearly displayed and will be very shortly cancelled by the macroscopic piece of solid, acting as a thermostat.

Low-temperature narrow ZPLs are very sensitive to various kinds of even very small irregularities of the surrounding solid, leading for a body of impurities to large inhomogeneous broadening of the ZPL. Depending on the solid host, on the impurity, and on the features of the electronic transition, the inhomogeneous ZPL width can vary from a tenth of $\mathrm{cm}^{-1}$ (e.g., rare-earth ions in good single crystals) to thousands of $\mathrm{cm}^{-1}$ (impurity molecules in amorphous glasses, frozen solutions, and polymers). Due to inhomogeneous broadening, even the narrowest low-temperature ZPL obtained by conventional absorption-emission measurements (i.e., without special spectroscopic techniques) comprises thousands of slightly different homogeneous ZPLs.

In the early years of ZPL spectroscopy [1,5], inhomogeneous broadening (IB) of optical spectra was considered as an annoying impediment, and the absence of IB in Mössbauer ZPLs was viewed as a great advantage of the latter. Nowadays the situation has changed [2,3]. IB of optical ZPLs has turned out to be a valuable feature for ZPL science and applications, especially in areas such as optical data storage and processing. In fact, the larger the ratio between the inhomogeneous linewidth $\left(\Gamma_{\text {inh }}\right)$ and the homogeneous linewidth $\left(\Gamma_{\text {hom }}\right), \alpha_{i h}=\Gamma_{\text {inh }} / \Gamma_{\text {hom }}$, the larger is the potential utility of these applications.

A ratio $\alpha_{i h}$ as high as $10^{8}$ has been achieved for $\mathrm{Er}^{3+}: \mathrm{LiNO}_{3}$ [19]. IB helps also to address a single molecule in SMS via spectrally selective excitation.

\section{Single impurity molecule spectroscopy (SMS) and single molecule detection (SMD)}

SMS and SMD (see [21], references therein and [22-25]) is a rapidly growing novel field of ZPLs studies. The applications are especially promoting in biology and molecular biology, also in chemistry [6]. A brand new development in physics are attempts to push ahead quantum computing, quantum communication, etc. Utilization of the sharp and narrow ZPLs $[1,2,16,21-23]$ for realization and transformation of qubits $(\mathrm{N}-\mathrm{V}$ centers in diamond) and also studies of single impurity molecules as single-photon sources and interference phenomena in SMS should be mentioned.

Both SMS and SMD are based on multiple excitation of luminescence and collecting photons. SMD can use also markers without ZPLs. On the other hand, the single impurity molecule spectroscopy, which is based on ZPLs and consequently requires low temperatures, is $3-5$ orders of more sensitive than SMD (the peak absorption cross section of fluorescence excitation being up to $5 \cdot 10^{-11} \mathrm{~cm}^{2}$ [24]). The shape of ZPL and other fine features can be measured. The peak absorption cross section and the spectral selectivity of luminescence excitation in SMD without ZPLs is $4-5$ orders of magnitude smaller than in SMS using good ZPLs. On the other hand, SMD also works at room temperatures, and this is a great advantages in biological studies.

A ZPL of $10 \mathrm{MHz}$ width (typical for the popular SMS molecular impurities today $[2-4,16])$ can be shifted to a new spectral position (compared to the ZPL's width) by a change of the host structure or a change of the electronic state of a neighboring impurity even hundreds of nanometers away. Besides caus- 
ing IB, this makes SMS a supersensitive method using ZPL impurities as probes. For it is sometimes too sensitive. The measurements of the widths of individual ZPLs in SMS reveal clearly the presence of various spectral diffusion processes even at liquid He temperatures [25-27]. An effective driving force at low temperatures is the nonequilibrium lattice energy stored in the cooling process. Measurements performed at various times after cooling of the sample display changes in the ZPLs' positions and shapes, remarkably different (individualistic) for different molecules. In most cases this is the result of the dependence of the IB on time [26,27].

A recent development which is promising, in particular, for fundamental quantum optics is the study of interference between the exciting laser beam and the resonantly scattered light emitted by a single molecule [28], and also the interference of emission of a single molecule with its mirror image [29]. The novel field of single-photon sources and single-photon phenomena has been opened.

Single-photon states of light today take an important position in quantum computation [30], quantum cryptography [31], and interferometric quantum nondemolition measurements [32]. Several candidates for single-photon sources has been proposed: faint laser pulses and parametric down-conversion [33], recombination of electrons and holes in single quantum dots [34], and optically excited single molecules [35].

The advantage of a single molecule as a source of single-photons is the clear situation that after emission of a photon, the emission of the next can take place only when the molecule gets excited again.

It takes some waiting time $T_{w}=T_{\text {exc }}+T_{\text {rad }}$, where $T_{\text {exc }}$ is the time required for excitation and $T_{\text {rad }}$ is the radiative lifetime, which is about $10^{-7} \mathrm{sec}$ for allowed transitions but can be orders of magnitude longer for forbidden ones. $T_{\text {exc }}$ is longer when excitation is weaker and the time gap between two subsequent photons longer. In interference experiments like [24] the excitation must match in good resonance the frequency of the ZPL, i.e., the $0-0$ transition. To have the possibility for this absorption, the vibrational relaxation in the ground electronic state must be completed. We have $T_{w}=T_{\text {exc }}+T_{\text {rad }}+T_{\text {rel }}$, where $T_{\text {rel }}$ is the (vibrational) relaxation time, which also can be very different for different impurity centers. Thus $T_{w}$ can be different by orders of magnitude and manipulated via excitation intensity.

A promising candidate for a photon gun and single-photon interference experiments is the $\mathrm{N}-\mathrm{V}$ impurity center (substitutional nitrogen trapped by a vacancy) in diamond [36,37], which is photostable even at room temperatures. First, the quality of the transi- tion of the $\mathrm{N}-\mathrm{V}$ center excited via the $\mathrm{ZPL}$ was verified to be good through measurements of the interphoton time in the process of photon counting and also through the second-order correlation functions of the fluorescence intensity. Both show clearly the antibunching nature of the process of photon emission and, consequently, that the source really is a single impurity center, emitting photons one by one separated by rather long time delays. To create and measure the interference, the stream of (single, well-separated in time and space) photons was directed into a Michelson interferometric detection set, and the change of the interference pattern of a photon with itself as a function of the delay introduced by the interferometer was detected. The measurements showed that in a system with an interferometer a single photon from a $\mathrm{N}-\mathrm{V}$ center exists as a superposition of two states of the single photon using both paths of the interferometer. The coherence length of the single-photon emitter was found from the decay of the visibility of the fringes in the interferogram. Interpretation of the measurements gave values in reasonable accordance with the expected ones for wavelength, linewidth, decay time (phase relaxation time $T_{2}$ ), and coherence length. When only the spectral interval corresponding to the ZPL frequency was selected for detection, the coherence length was increased by more than two orders of magnitude.

SMS has been successfully applied to spin dynamics in organic molecules [38-40] and proposed for sequencing of nucleotides in the DNA molecule $[41,42]$. Let us point out that the task of sequencing of nucleotide in the DNA molecule is an ideal one for low-temperature SMS spectroscopy: the biological essence the sequence - is most probably not spoiled by cooling to liquid He and applying moderate laser excitation.

Recently single molecule spectroscopy has been applied to paramagnetic single nitrogen-vacancy $(\mathrm{N}-\mathrm{V})$ defect centers in diamond [43-48] (actually the only SMS-studied inorganic impurity center up to now). They show high fluorescence emission yield, allowing optical detection of individual defects at low temperature under resonant excitation. The measured homogeneous linewidth of $150 \mathrm{MHz}$ is smaller than the $2.88 \mathrm{GHz}$ zero-field splitting of the triplet ground state of the center, allowing the control of the single electron spin state [8]. Individual spin dynamics has been analyzed qualitatively using fluorescence correlation spectroscopy [49]. Time-resolved data show that spin-lattice relaxation time is in the range of milliseconds.

It was shown that polarization-sensitive single-molecule fluorescence analysis provides a detailed insight into the photophysics of photosynthetic antenna com- 
plexes. The picture of excitonic interactions in the light-harvesting complex LH2 is a matter of significant perturbation at room temperature. Dynamical disorder results in the fluctuation of the emission polarization on a time scale of a few hundreds of milliseconds [50]. Strong influence of the local protein environment on the spectral characteristic of pigments was observed for plant antenna LHCII [51]. This photosynthetic aggregate was extensively investigated at low temperature, where the spectral jumps of individual Chls molecules reveal the dynamics of the protein matrix. Room temperature studies of LHCII show that only one emitting state is responsible for the fluorescence emission at room temperature. For the trimeric LHCII, the energy transfer between monomeric subunits is slower than the fluorescence lifetime, resulting in an unpolarized fluorescence emission. Recently the single-molecule approach was successfully applied to a reaction center containing a photosynthetic aggregate - Photosystem I from the cyanobacterium Synechococcus elongatus [52,53]. It was shown that the red-most pigment pool contains two subgroups of Chls molecules, which differ not only by their spectral position but also by the strength of the electron-phonon coupling. It was proposed that the red-most state is a Chl dimer, which is located near the special pair. High-resolution fluorescence emission and excitation spectra revealing the single chlorophyll structure were recorded at cryogenic temperatures. It was shown that the low fluorescence quantum yield (a few percent at room temperature) is not a severe obstacle to detection of the reaction center containing the photosynthetic species. Quenching of pigment fluorescence can be deactivated at cryogenic temperatures when the required uphill energy transfer is blocked.

The homogeneous width $\Gamma_{\text {hom }}$ (shape) of the ZPL is important especially in the novel applications, and to have its definition seems desirable. If the measurement of luminescence (of secondary emission, generally speaking) is performed shortly after the act of excitation with a short pulse, then the result is a time-dependent spectrum which can differ entirely from those of conventional stationary measurements. The critical time parameters are the radiative lifetime and the nonradiative relaxation times. If the measurement period is too long, then spectral diffusion can introduce basic changes. Therefore, I would suggest the following definition of the homogeneous ZPL's line shape: «the homogeneous ZPL spectrum is that of a single impurity center measured in a proper time interval» [54].

For sake of quantitative interpretation of experimental data, it is generally useful to consider four se- parate factors that can contribute to the width of homogeneous ZPL (and also the line shape):

$$
\begin{aligned}
& \Gamma_{\text {hom }}(T, t)=\Gamma_{\text {rad }}(T)+\Gamma_{\text {dep }}(T)+ \\
& +\Gamma_{\text {nrad }}(T)+\Gamma_{\text {inh }}(T, t)
\end{aligned}
$$

where $\Gamma_{\operatorname{rad}}(T)$ is the linewidth caused by spontaneous emission (for allowed transitions the $T$ dependence is not critical); $\Gamma_{\text {nrad }}(T)$ is the linewidth caused by quenching of luminescence (the $T$ dependence is critical); $\Gamma_{\text {dep }}(T)$ is the line broadening caused by dephasing, in most cases by scattering of phonons at the impurity, leading to degradation of the phase coherence (optical coherence) of the excited electronic state (the $T$ dependence is strong because the number of phonons in the solid increases rapidly with $T$ ); $\Gamma_{\text {inh }}(T, t)$ arises from different rearrangements and heating/cooling processes taking place in the matrix during the measurement. For individual single molecules the time dependence of the inhomogeneous broadening is also individual and can be strong and complicated. For example, in SMS hundreds of fluorescence photons are being collected for each point of the spectrum, and the measurement process may last up to tens of seconds.

Note that every individual impurity may have its own values for each of the factors in (1). Linewidth SMS measurements of individual molecules have revealed variations of $\Gamma_{\text {hom }}(T, t)$ up to tens of percent between chemically identical impurity centers and also from one measurement time to another delayed by tens of seconds (see next Section). Variations in $\Gamma_{\text {rad }}$ may occur; the dependence of $\Gamma_{\text {rad }}$ can be due to different densities of photon states in the crystal and in vacuum, or can depend on its position relative to the sample's surface. It is interesting to note that similar dependence on the density of the electromagnetic field states causes the well-known Casimir effect [55].

Persistent spectral hole burning (PSHB) is actually bleaching of luminescence. What makes PSHB a remarkable and useful phenomenon is that the bleaching can be performed, owing to ZPLs, with very high spectral selectivity - with an accuracy of their width. A precious gift of Nature is that the very narrow homogeneous ZPLs form a broad inhomogeneous ZPL band. Illuminating a PSHB material by a light beam having the intensity spectrum (summed up over the excitation time) $I(\omega)$ bleaches the inhomogeneous absorption in accordance with $I(\omega)$ and forms «a hole» having the shape $I(\omega)$ and thus memorizing the frequency distribution of the excitation. PSHB is an effective tool of optical engineering by means of illumination: it enables one to predesign and change the profile of the inhomogeneous absorption band of impurity absorption, i.e., actually to control the coeffi- 
cient of absorption and the index of refraction, which is bound to it via the Kramers-Kronig relations. When we deal with time-dependent holographic pictures (events) (see next Section) this relation guarantees causality in the theoretical description. A novel chapter of optical engineering has opened using light as the tool. The hole structure fixed in the changes of the sample's material can have long and very long lifetimes - depending on the lifetime of the changes caused by bleaching. In [56] the lifetime of holes in deuterated organic solids has been estimated as a hundred thousand years. Thus PSHB devices can be made: broad-spectral-band and broad-apperture spectral filters, modulators and, the most remarkable application - optical memories having lifetimes lasting hours, days, and longer.

In the case of pulsed light excitation, the intensity spectrum of the bleaching pulse is stored in the broad and complicated hole structure with a high accuracy equal to the width $\Gamma_{\mathrm{hom}}$. If the approach of holography is applied - a reference pulse (shifted in time) is added to the pulse carrying the information and the two pulses are stored together - the equivalent of phases of the information pulse's Fourier components can also be stored. Thus the signal pulse can be restored completely and with high accuracy. The realization of this possibility has opened new and spectacular chapters of optical information storage and processing - frequency-and-space-domain holography [57] and time-and-space-domain holography (TSDH) (see $[3,4,17,58]$ and references therein). If the excitation laser band is narrow compared to the homogeneous linewidth $\Delta \omega_{l} \ll<\Gamma_{\text {hom }}$ the hole width measured (in the limit of weak excitation) is $\Gamma_{\text {hol }}=2 \Gamma_{\text {hom }}$. Factor 2 originates from $\Gamma_{\text {hom }}$ being involved twice first, in hole burning and, second, in the hole shape measurement.

The selectivity, given by $\Gamma_{\text {hom }} \approx 10^{-3} \mathrm{~cm}^{-1}$, shows that the profile of a picosecond pulse (with $\Delta \omega_{\mathrm{exc}} \approx 10$ $\mathrm{cm}^{-1}$ ) can be stored with a rather high accuracy, given by the ratio

$$
\Delta_{\text {exc }}: \Gamma_{\text {hom }} \approx 10: 10^{-3} \approx 10^{4} .
$$

Here $\Gamma_{\text {inh }}>10 \mathrm{~cm}^{-1}$.

The remarkable feature of PSHB holographic studies and applications is that practically everything proposed theoretically has been realized experimentally the laboratory environment. Commercial applications are still absent because the really nice applications require large $\alpha$ and, consequently, also low temperatures. In fact, liquid He temperatures are nowadays quite a minor technical problem. An essential recent development is femtosecond PSHB.
Femtosecond pulses. The pulses of 10-100 fs duration and hundreds of $\mathrm{cm}^{-1}$ wide provide a convenient field for frequency-domain experiments and thus also PSHB technique. The first femtosecond experiment was published ten years ago [59]. In [60] it is shown how to produce arbitrary time-domain pulse shapes by using PSHB.

The Fourier amplitudes of the desired time dependence of the light pulse are calculated. The corresponding profile of the frequency dependence of the absorption coefficient of a PSHB sheet is written in through PSHB by scanning a narrow line laser.

The PSHB sheet prepared in that way is illuminated with a broad band pulse (a «white pulse») which, after passing through, is modulated in accordance with the calculated profile of the absorption coefficient (together with it the index of refraction) and will propagate having the desired shape in time [60].

If a time-dependent picture (an event) is to be composed (a scene of an artificial movie) the procedure has to be performed for each spatial pixel of the PSHB sheet.

In the case of a given time-dependent picture (event) it is projected to the sheet. When the PSHB sheet prepared in that way is illuminated with a properly directed «white» interrogating pulse, an image of the event is created in full: color picture, time dependence, and even polarization properties are available. The same PSHB structure can be created pixel-bypixel by means of a cw laser writing the precalculated hole structure. Note that the scenes are artificial: nothing like them ever existed (happened) in reality. The time dependence is continuous, different from our conventional movies or TV, where it is a deception created by changing static pictures with $50 \mathrm{~Hz}$ frequency. PSHB time-and-space-domain holography provides a means for really continuous time dependence. In principle, to make a movie, only the scenario, a team of PSHB laser-optics professions and proper equipment and materials are necessary. No artists, no requisites, panoramic sights, etc. are needed. The present-day limitation comes from the materials - the maximum duration of a scene is limited by the material's phase relaxation time to small fractions of second.

In [61] a femtosecond interference effect created in a ultrafast two-pulse photon echo in dye-doped polymer films at low temperatures was used to demonstrate an ultrafast three-port all-optical logic «controlled NOT» gate (Taffoli gate).

A fascinating result of nonlinear optics is the very large slowing down of the group velocity of light in matter down to the complete «stopping of light» (see $[62,63]$ and references therein). In fact it means that 
the information carried by a light pulse can be stored in the structure of $n(x)$ and later (within the limits of the coherence time of the excited state of matter), be retrieved by a suitable interrogating pulse. PSHB time-and-space domain holography has performed the same task with the following essential differences: coherence (no phase relaxation of the excited state is required only for the write-in time), and the storage time can, as mentioned above, last hours and if desired, be even longer by orders of magnitude. Note that the light-matter interaction in PSHB is linear [3,4,64].

An interesting brand new attempt of indicate the possibility of considerably increasing (by a factor of $\sim 100$ ) the number of qubits involved in processing is made via using spectral holes [65].

\section{Promises of quantum computing compared to what is done in PSHB holography}

What can be actually achieved in quantum computing (QC) is not clear yet. Current literature prefers to focus on comparing the future of $\mathrm{QC}$ to the present state of conventional digital electronic data storage and computing. The outcome of such comparison is overwhelmingly in favor of the future QC. However, it would be fair and educational if QC would be also compared to holographic data storage and processing, in particular, to PSHB-based time-and-space-domain holography (see [66]).

Holography is based on distributed data storage and uses interference of light waves. PSHB adds to the two pillars: the frequency-time dimension. In holography, owing to these features, the parallelism in storage, processing and retrieval of information is naturally present. Holography provides self-correction of errors and associative recall of information, PSHB $\mathrm{TSDH}$ - also in the time domain - in the sequence of events; effective data search (search for events), retrieval of the whole picture (event) by interrogating with a fragment of the picture (event). Time-andspace-domain holography comprises the time-arrow and allows manipulating temporal order of recalled signals, time-reversal, time lens and fast time shutter (based on causality) [2-4,64].

As a further advantage of holography I would like to point out that it is based on read-out of whole pictures and events, which appears as a natural path to human comprehension. In contrast, digital computers, no matter how advanced they may get in the future, produce «mountains of bits», which are digestible only for another large computer.

The strongest point of TSDH compared to QC is that phase memory has to be preserved only for the time of write-in. In the experiments performed up to now the phase memory of the excited electronic states lasts for about $1 \mathrm{~ns}$ in organic materials and up to $1 \mathrm{~ms}$ in rare-earth doped crystals. After the phase information is written in, it will be preserved through the stored pattern of intensities (amplitudes) in the modulated structure of the complex index of refraction $n(r, \omega)$ The latter is fixed by the rather stable positions of the atoms of the sample. The information can be read out at a later time (up to years) and many times over.

In contrast, $\mathrm{QC}$ requires that phase memory be preserved at all times during writing, storing, and readout of the information. The last completely destroys the content of the memory. It would be desirable to combine the ideas of $\mathrm{QC}$ with the features of holography (or to prove that such a combination is impossible).

\section{Conclusion}

The laser era of ZPL studies and applications started almost 40 years ago, persistent spectral hole burning is thirty years old and single-impurity-molecule spectroscopy ten years of age. All three are in good health, producting and promising fine new results. The most interesting results and future developments seem to lie in optical data storage and processing, including quantum informatics. We have to expect remarkable results also in SMS and SMD applications through single marker-carrying impurities as probes in biology.

The author is grateful to F. Jelezko, A. Rebane, and J. Wrachtrup for discussions (especially to F.J. for information and references on SMS studies on $\mathrm{N}-\mathrm{V}$ centers in diamond and photosynthetic units); to the A. von Humboldt Foundation for making possible my work at Stuttgart University, and to the World Laboratory and Estonian Science Foundation (grant 4003) for support.

1. K. Rebane, Impurity Spectra of Solids, Plenum Press, New York (1970).

2. Zero-Phonon Lines and Spectral Hole Burning in Spectroscopy and Photochemistry, O. Sild and K. Haller (eds.), Springer, Berlin (1988).

3. K.K. Rebane and A. Rebane, in: Molecular Electronics, Properties, Dynamics and Applications, G. Mahler, V. May, and M. Schreiber (eds.), Marcel Dekker, New York (1996), Chapter 13, p. 257.

4. K.K. Rebane, in: Current Trends in Optics, J.C. Dainty (ed.), Academic Press, London (1994), Chapter 13 , p. 177.

5. K.K. Rebane and V.V. Hizhnyakov, Opt. Spectrosc. 14, 362 (1963); ibid. 14, 491 (1963).

6. E.D. Trifonow, Dokl. Akad. Nauk USSR 147, 826 (1962). 
7. E.F. Gross, B.S. Razbirin, and S.A. Permogorov, Dokl. Akad. Nauk SSSR 147, 338 (1962); E.F. Gross, S.A. Permogorov, and B.S. Razbirin, ibid. 154, 1306 (1964).

8. M.A. Krivoglaz and S.I. Pekar, Trudy Instituta Fiziki AN Ukr. SSR, Kiev 4, 37 (1953).

9. R. Mössbauer, Zs. Physik 151, 124 (1958); Zs. Naturforschung 14a, 211 (1959).

10. Spectroscopy of Solids Containing Rare-Earth Ions. Modern Problems in Condensed Matter Science, A.A. Kaplyanskii and R.M. Macfarlan (eds.), vol. 21, NorthHolland (1989).

11. A.L. Schawlow, in: Advances in Quantum Electronics, J.R. Singer (ed.), New York-London (1961), p. 50; G.F. Imbusch, W.M. Yen, A.L. Schawlow, D.E. McCumber, and M.D. Struge, Phys. Rev. A133, 1029 (1964).

12. L.A. Rebane, A.A. Gorokhovskii, and J.V. Kikas, J. Appl. Phys. B29, 215 (1982).

13. A. Szabo, Phys. Rev. Lett. 25, 924 (1970).

14. L.A. Rebane, Zhurnal Prikladnoi Spektroskopii 34, 1023 (1981) (in Russian).

15. Karl K. Rebane, J. Chem. Phys. 189, 139 (1994).

16. T. Basche, W.E. Moerner, M. Orrit, and U.P. Wild, Single-Molecule Optical Detection, Imaging and Spectroscopy, Weinheim, New York (1997).

17. Persistent Spectral Hole Burning, Science and Applications, W.E. Moerner (ed.), Springer, Berlin (1988).

18. R.W. Equall, Y. Sun, R.L. Cone, and R.M. Macfarlane, Phys. Rev. Lett. 72, 2179 (1994).

19. Y. Sun, C.W. Thiel, R.L. Cone, R.W. Equall, and R.I. Hutcheson, J. Lumin. 98, 281 (2002).

20. O. Sild and K. Rebane, Opt. Spectrosc. (St. Petersburg) 90, 766 (2001) (in Russian) [90, 686 (2001) (in English)].

21. F. Güttler, Spektroskopie Einzelner Moleküle, Dissertation ETH nr. 10707, Zürich (1994).

22. W.E. Moerner and L. Kador, Phys. Rev. Lett. 62, 2535 (1989).

23. M. Orrit and J. Bernard, Phys. Rev. Lett. 65, 2716 (1990).

24. K. Rebane and I. Rebane, J. Lumin. 56, 39 (1993).

25. V. Palm, K. Rebane, and A. Suisalu, J. Phys. Chem. 98, 2219 (1994).

26. K.K. Rebane, O. Ollikainen, and V.V. Palm, Opt. Spectrosc. (St. Petersburg) 84, 431 (1998) (in Russian) [84, 374 (1989) (in English)].

27. V.V. Palm and K.K. Rebane, Phys. Solid State (St. Petersburg) 42, 477 (2000).

28. T. Plakhotkin and V. Palm, Phys. Rev. Lett. 87, 183602 (2001).

29. J. Escher, Ch. Raab, F. Schmidt-Kaier, and R. Blatt, Nature 413, 496 (2001).

30. E. Knill, R. Laflamme, and G.J. Milburn, Nature 409, 46 (2001).

31. N. Gisin, G.G. Ribordy, W. Tittel, and H. Zbinden, Rev. Modern Physics 74, 145 (2002).

32. B. Lounis and W.E. Moerner, Nature 407, 491 (2000).

33. G. Nogues et al., Nature 400, 239 (1999).
34. P. Michler et al., Science 290, 2282 (2000); A. Peveratos, R. Brouri, T. Gacoin, J.P. Poizat, and P. Grangier, Phys. Rev. A6406, 061802 (2001).

35. A. Gruber et al., Science 276, 2012 (1997).

36. F. Jelezko, A. Volkmer, I. Popa, K.K. Rebane, and J. Wrachtrup (submitted).

37. V. Kiisk, V. Palm, A. Suisalu, and I. Sildos, J. Lumin. 86, 349 (2000); V. Hizhnyakov, H. Kaasik, and I. Sildos, Phys. Status Solidi B234, 644 (2002).

38. J. Wrachtrup, C. von Borczyskowski, J. Bernard, M. Orrit, and R. Brown, Nature 363, 244 (1993).

39. J. Wrachtrup, C. von Borczyskowski, J. Bernard, M. Orrit, and R. Brown, Phys. Rev. Lett. 71, 3565 (1993).

40. R. Brown, J. Wrachtrup, M. Orrit, J. Bernard, and C. von Borczyskowski, J. Chem. Phys. 100, 7182 (1994).

41. K.K. Rebane, Experimental Technique of Physics 41, 295 (1995); Solid State Physics (St. Petersburg) 38, 3487 (1996) (in Russian); ibid. 38, 1902 (1996).

42. K. Rebane, A Means and Method for Sequencing Nucleotides in DNA Molecule Using Laser Spectroscopy, Patent application of Tart University, accepted in Estonian Patent Agency 27.09.96, No 0134196; No P 9600101, 15.04.98.

43. A. Gruber, A. Drabenstedt, C. Tietz, L. Fleury, J. Wrachtrup, and C. von Borczyskowski, Science 276, 2912 (1997).

44. A. Drabenstedt, C. Tietz, F. Jelezko, J. Wrachtrup, S. Kilin, and A.Nizovtzev, Acta Physica Polonica A 96, 665 (1999).

45. A. Drabenstedt, L. Fleury, C. Tietz, F. Jelezko, S. Kilin, A. Nizovtzev, and J. Wrachtrup, Phys. Rev. B60, 11503 (1999).

46. F. Jelezko, C. Tietz, A. Gruber, I. Popa, A. Nizovtsev, S. Kilin, and J. Wrachtrup, Single Molecules 2, 255 (2001).

47. F. Jelezko, I. Popa, A. Gruber, C. Tietz, J. Wrachtrup, A. Nizovtsev, and S.Kilin, Appl. Phys. Lett. 81, 2160 (2002).

48. S.Y. Kilin, A.P. Nizovtsev, T.M. Maevskaya, A. Gruber, A. Drabenschtedt, J. Wrachtrup, and C. von Borczyskowski, Opt. Spectrosc. 87, 624 (1999).

49. A.P. Nizovtsev, S.Y. Kilin, C. Tietz, F. Jelezko, and J. Wrachtrup, Physica B308, 608 (2001).

50. C. Tietz, O. Chekhlov, A. Drabenstedt, J. Schuster, and J. Wrachtrup, J Phys. Chem. B103, 6328 (1999).

51. C. Tietz, F. Jelezko, U. Gerken, S. Schuler, A. Schubert, H. Rogl, and J. Wrachtrup, Biophys. J. 81, 556 (2001).

52. F. Jelezko, C. Tietz, U. Gerken, J. Wrachtrup, and R. Bittl, J. Phys. Chem. B104, 8093 (2000).

53. F. Jelezko, C. Tietz, U. Gerken, E. Thews, S. Schuler, A. Wechsler, and J. Wrachtrup, Opt. Spectrosc. 91, 457 (2001).

54. K. Rebane, J. Lumin. 100, 219 (2002).

55. H.B.G. Casimir and D. Polder, J. Phys. (Paris) 46, 407 (1949); H.B.G. Casimir and D. Polder, Phys. Rev. 73, 360 (1948). 
56. J. Friedrich and D. Haarer, Angew. Chemie 23, 113 (1984); D. Haarer, in [17] p. 79.

57. A. Renn, U.P. Wild, and A. Rebane, J. Phys. Chem. 106, 3045 (2002); C.D. Caro, S. Bernet, A. Renn, and U.P. Wild, in: Molecular Electronics, Properties, Dynamics and Applications, Marcel Dekker, New York (1996), Ch. 14, p. 303.

58. A. Rebane, Cimia 52, 112 (1998).

59. A. Rebane, J. Aaviksoo, and J. Kuhl, Appl. Phys. Lett. 54, 93 (1989).

60. A. Rebane, M. Drobizhev, and C. Sigel, Opt. Lett. 25, 1633 (2000).

61. M. Drobizhev, A. Karotki, and A. Rebane, Chem. Phys. Lett. 334, 76 (2001)
62. B. G. Levi, Physics Today, March 2001, p. 17.

63. D.E. Phillips, A. Fleischhauer, A. Mair, R.L. Walsworth, and M.D. Lunin, Phys. Rev. Lett. 86, 783 (2001).

64. A. Rebane, in: Trends in Optics. Research Developments and Applications, A. Consortini (ed.), San Diego, Academic Press (1996), p. 165.

65. M.S. Shahriar, P.R. Hemmer, S. Lloyd, P.S. Bathia, and A.E. Craig, Phys. Rev. A66, 032301 (2002).

66. K.K. Rebane, Opt. Spectrosc. (St. Petersburg) 91 472 (2001) 